\title{
Existence of positive solutions for $p$-Laplacian impulsive boundary value problems on time scales
}

Fatma Tokmak ${ }^{1,2}$ and Ilkay Y Karaca ${ }^{*^{*}}$

${ }^{*}$ Correspondence:

ilkay.karaca@ege.edu.tr

${ }^{1}$ Department of Mathematics, Ege

University, Bornova, Izmir, 35100,

Turkey

Full list of author information is

available at the end of the article

\begin{abstract}
In this paper, by using fixed point index theory and a double fixed point theorem, we study the existence of many positive solutions for a class of second-order $p$-Laplacian boundary value problems with impulse on time scales. An example which supports our theoretical results is also indicated.
\end{abstract}

MSC: 34B18; 34B37; 34K10

Keywords: multiple positive solutions; double fixed point theorem; fixed point index; time scales; impulsive boundary value problems

\section{Introduction}

It is well known that the theory of impulsive differential equations has become more important in recent years in some mathematical models of real processes and phenomena. For the introduction of the basic theory of impulsive equations, see [1-4] and the references therein.

The theory of dynamic equations on time scales has been developing rapidly and has received much attention in recent years. The study unifies existing result in differential and finite difference equations and provides powerful new tools for exploring connections between the traditionally separated fields. We refer to the books by Bohner and Peterson $[5,6]$.

However, there is not so much work on the impulsive boundary value problem with $p$-Laplacian on time scales except that in [7-11]. The authors studied impulsive dynamic equations on time scales without $p$-Laplacian in [12-15] (see also references therein), while in [16-18] the authors considered $p$-Laplacian impulsive differential equations without time scales. The authors considered impulsive boundary value problems without $p$-Laplacian and time scales in $[19,20]$.

Motivated by the above results, in this study we consider the following second-order impulsive boundary value problem (BVP) on time scales:

$$
\begin{aligned}
& -\left[\phi_{p}\left(u^{\Delta}(t)\right)\right]^{\nabla}=f(t, u(t)), \quad t \in J:=[0,1]_{\mathbb{T}}, t \neq t_{k}, k=1,2, \ldots, m, \\
& -\left.\Delta u\right|_{t=t_{k}}=I_{k}\left(u\left(t_{k}\right)\right), \\
& u^{\Delta}(0)=0, \quad \alpha u(1)+\beta u^{\Delta}(1)=\int_{0}^{1} g(s) u(s) \Delta s,
\end{aligned}
$$

O2014 Tokmak and Karaca; licensee Springer. This is an Open Access article distributed under the terms of the Creative Commons Attribution License (http://creativecommons.org/licenses/by/2.0), which permits unrestricted use, distribution, and reproduction in any medium, provided the original work is properly cited. 
where $\mathbb{T}$ is a time scale, $0,1 \in \mathbb{T}, J=[0,1]_{\mathbb{T}}=[0,1] \cap \mathbb{T}, t_{k} \in(0,1)_{\mathbb{T}}, k=1,2, \ldots, m$ with $0<$ $t_{1}<t_{2}<\cdots<t_{m}<1, \alpha>\int_{0}^{1} g(s) \Delta s, \beta>0, \phi_{p}(s)$ is a $p$-Laplacian operator, i.e., $\phi_{p}(s)=|s|^{p-2} s$, $p>1,\left(\phi_{p}\right)^{-1}=\phi_{q}, \frac{1}{p}+\frac{1}{q}=1,\left.\Delta u\right|_{t=t_{k}}$ denotes the jump of $u(t)$ at $t=t_{k}$, i.e.,

$$
\left.\Delta u\right|_{t=t_{k}}=u\left(t_{k}^{+}\right)-u\left(t_{k}^{-}\right)
$$

where $u\left(t_{k}^{+}\right)=\lim _{h \rightarrow 0^{+}} u\left(t_{k}+h\right)$ and $u\left(t_{k}^{-}\right)=\lim _{h \rightarrow 0^{+}} u\left(t_{k}-h\right)$ represent the right-hand limit and left-hand limit, respectively, of $u(t)$ at $t=t_{k}$. In addition, $f, I_{k}$, and $g$ satisfy

(H1) $f \in \mathcal{C}\left([0,1]_{\mathbb{T}} \times[0,+\infty),[0,+\infty)\right)$

(H2) $I_{k} \in \mathcal{C}([0,+\infty),[0,+\infty))$;

(H3) $g \in \mathcal{C}_{r d}\left([0,1]_{\mathbb{T}},[0,+\infty)\right)$ is nonnegative.

We remark that by a solution $u$ of (1.1)-(1.3), we mean $u: \mathbb{T} \rightarrow \mathbb{R}$ is delta differentiable, $u^{\Delta}: \mathbb{T}^{k} \rightarrow \mathbb{R}$ is nabla differentiable on $\mathbb{T}^{k} \cap \mathbb{T}_{k}$ and $u^{\Delta \nabla}: \mathbb{T}^{k} \cap \mathbb{T}_{k} \rightarrow \mathbb{R}$ is continuous, and it satisfies the impulsive and boundary value conditions (1.2)-(1.3). If $u^{\Delta \nabla} \leq 0$ on $[0,1]_{\mathbb{T}^{k} \cap \mathbb{T}_{k}}$, then we say that $u$ is concave on $[0,1]_{\mathbb{T}}$.

By using the fixed point index theory in the cone [21], we get the existence of at least two positive solutions for the impulsive BVP (1.1)-(1.3). Then we generalized this to obtain many positive solutions. By using a double fixed point theorem [22], we also establish the existence of countably many positive solutions for the impulsive BVP (1.1)-(1.3). To the best of our knowledge, no paper has considered the second-order impulsive BVP with integral boundary conditions and one dimensional $p$-Laplacian on time scales. In fact, our results are also new when $\mathbb{T}=\mathbb{R}$ (the differential case) and $\mathbb{T}=\mathbb{Z}$ (the discrete case). Therefore, the results can be considered as a contribution to this field.

This paper is organized as follows. In Section 2, we provide some necessary background about time scales, the theory of cones in Banach space and some preliminary lemmas. We give and prove our main results in Section 3. Finally, in Section 4, we give an example to demonstrate our results.

\section{Preliminaries}

In this section, we present some definitions and theorems, which will be needed in the proof of the main results.

Definition 2.1 Let $(\mathbb{B},\|\cdot\|)$ be a real Banach space. A nonempty closed set $K \subset \mathbb{B}$ is said to be a cone provided that

(i) $a x+b y \in K$ for all $x, y \in K$ and $a, b \geq 0$;

(ii) $y,-y \in K$ implies $y=0$.

Every cone $K \subset \mathbb{B}$ induces an ordering in $\mathbb{B}$ given by $x \leq y$ if and only if $y-x \in K$.

Definition 2.2 The map $\alpha$ is said to be a nonnegative continuous concave functional on a cone $K$ of a real Banach space $\mathbb{B}$, provided that $\alpha: K \rightarrow[0, \infty)$ is continuous and

$$
\alpha(t u+(1-t) v) \geq t \alpha(u)+(1-t) \alpha(v)
$$

for all $u, v \in K, 0 \leq t \leq 1$. 
Similarly, we say that the map $\gamma$ is a nonnegative continuous convex functional on a cone $K$ of a real Banach space $\mathbb{B}$ provided that

$$
\gamma(t u+(1-t) v) \leq t \gamma(u)+(1-t) \gamma(v)
$$

for all $u, v \in K, 0 \leq t \leq 1$.

For a nonnegative continuous functional $\gamma$ on a cone $K$ in a real Banach space $\mathbb{B}$, and for each $d>0$, we set

$$
K(\gamma, d)=\{x \in K \mid \gamma(x)<d\}
$$

The following fixed point theorems are fundamental and important to the proofs of our main results.

Theorem 2.1 (Double fixed point theorem [22]) Let $K$ be a cone in a real Banach space $\mathbb{B}$. Let $\alpha$ and $\gamma$ be increasing, nonnegative, continuous functionals on $K$, and let $\theta$ be a nonnegative, continuous functional on $K$ with $\theta(0)=0$ such that, for some $c>0$ and $M>0$,

$$
\gamma(x) \leq \theta(x) \leq \alpha(x) \quad \text { and } \quad\|x\| \leq M \gamma(x)
$$

for all $x \in \overline{K(\gamma, c)}$. Suppose that there exist positive numbers $a$ and $b$ with $a<b<c$ such that

$$
\theta(\lambda x) \leq \lambda \theta(x), \quad \text { for } 0 \leq \lambda \leq 1 \text { and } x \in \partial K(\theta, b)
$$

and

$$
T: \overline{K(\gamma, c)} \rightarrow K
$$

is a completely continuous operator such that:

(i) $\gamma(T x)>c$, for all $x \in \partial K(\gamma, c)$;

(ii) $\theta(T x)<b$, for all $x \in \partial K(\theta, b)$;

(iii) $K(\alpha, a) \neq \emptyset$, and $\alpha(T x)>a$, for all $x \in \partial K(\alpha, a)$.

Then $T$ has at least two fixed points, $x_{1}$ and $x_{2}$ belonging to $\overline{K(\gamma, c)}$ such that

$$
a<\alpha\left(x_{1}\right) \text {, with } \theta\left(x_{1}\right)<b \text {, }
$$

and

$$
b<\theta\left(x_{2}\right), \text { with } \gamma\left(x_{2}\right)<c \text {. }
$$

Theorem 2.2 [21] Let $K$ be a cone in a real Banach space $\mathbb{B}$. Let $D$ be an open bounded subset of $\mathbb{B}$ with $D_{K}=D \cap K \neq \emptyset$ and $\overline{D_{K}} \neq K$. Assume that $T: \overline{D_{K}} \rightarrow K$ is completely continuous such that $x \neq T x$ for $x \in \partial D_{K}$. Then the following results hold:

(i) If $\|T x\| \leq x, x \in \partial D_{K}$, then $i_{K}\left(T, D_{K}\right)=1$.

(ii) If there exists $e \in K \backslash\{0\}$ such that $x \neq T x+\lambda$ efor all $x \in \partial D_{K}$ and all $\lambda>0$, then $i_{K}\left(T, D_{K}\right)=0$.

(iii) Let $U$ be open in $P$ such that $\bar{U} \subset D_{K}$. If $i_{K}\left(T, D_{K}\right)=1$ and $i_{K}\left(T, U_{K}\right)=0$, then $T$ has a fixed point in $D_{K} \backslash \overline{U_{K}}$. The same result holds if $i_{K}\left(T, D_{K}\right)=0$ and $i_{K}\left(T, U_{K}\right)=1$. 


\section{Main results}

In this section, by defining an appropriate Banach space and cone, we impose growth conditions on $f$ and $I_{k}$ which allow us to apply the theorems in Section 2 to establish the existence results of the positive solutions for the BVP (1.1)-(1.3).

Let $J^{\prime}=[0,1]_{\mathbb{T}} \backslash\left\{t_{1}, t_{2}, \ldots, t_{m}\right\}$. We define

$$
\begin{aligned}
\mathbb{B}= & \left\{u \mid u:[0,1]_{\mathbb{T}} \rightarrow \mathbb{R} \text { is continuous at } t \neq t_{k}, \text { left continuous at } t=t_{k},\right. \\
& \text { and there exist } \left.u\left(t_{k}^{-}\right) \text {and } u\left(t_{k}^{+}\right) \text {with } u\left(t_{k}^{-}\right)=u\left(t_{k}\right) \text { for } k=1,2, \ldots, m\right\} .
\end{aligned}
$$

Then $\mathbb{B}$ is a real Banach space with the norm $\|u\|=\sup _{t \in[0,1]_{\mathbb{T}}}|u(t)|$. By a solution of (1.1)(1.3), we mean a function $u \in \mathbb{B} \cap \mathcal{C}^{2}\left(J^{\prime}\right)$ which satisfies (1.1)-(1.3). We define a cone $K \subset \mathbb{B}$ as

$$
\begin{aligned}
K= & \left\{u \in \mathbb{B}: u \text { is a nonnegative and nonincreasing function, } u^{\Delta}\right. \text { is } \\
& \text { nonincreasing on } \left.J^{\prime}, \alpha u(1)+\beta u^{\Delta}(1)=\int_{0}^{1} g(s) u(s) \Delta s\right\} .
\end{aligned}
$$

We need the following lemmas that will be used to prove our main results.

Lemma 3.1 Suppose that $(\mathrm{H} 1)-(\mathrm{H} 3)$ are satisfied. Then $u \in \mathbb{B} \cap \mathcal{C}^{2}\left(J^{\prime}\right)$ is a solution of the impulsive boundary value problem (1.1)-(1.3) if and only if $u(t)$ is a solution of the following integral equation:

$$
\begin{aligned}
u(t)= & \frac{1}{\alpha-\int_{0}^{1} g(s) \Delta s} \int_{0}^{1} g(s)\left(\int_{s}^{1} \phi_{q}\left(\int_{0}^{\tau} f(r, u(r)) \nabla r\right) \Delta \tau\right) \Delta s \\
& +\frac{1}{\alpha-\int_{0}^{1} g(s) \Delta s}\left[\beta \phi_{q}\left(\int_{0}^{1} f(s, u(s)) \nabla s\right)\right. \\
& \left.+\int_{0}^{1} g(s)\left(\sum_{s \leq t_{k}} I_{k}\left(u\left(t_{k}\right)\right)\right) \Delta s\right]+\int_{t}^{1} \phi_{q}\left(\int_{0}^{s} f(r, u(r)) \nabla r\right) \Delta s \\
& +\sum_{t \leq t_{k}} I_{k}\left(u\left(t_{k}\right)\right),
\end{aligned}
$$

and $u(t) \geq 0$.

Proof Integrating (1.1) from 0 to $t$, one has

$$
-\phi_{p}\left(u^{\Delta}(t)\right)+\phi_{p}\left(u^{\Delta}(0)\right)=\int_{0}^{t} f(s, u(s)) \nabla s .
$$

By the boundary condition (1.3), we have

$$
u^{\Delta}(t)=-\phi_{q}\left(\int_{0}^{t} f(s, u(s)) \nabla s\right)
$$


Integrating the dynamic equation above from $t$ to 1 , we get

$$
u(t)=u(1)+\int_{t}^{1} \phi_{q}\left(\int_{0}^{s} f(r, u(r)) \nabla r\right) \Delta s+\sum_{t \leq t_{k}} I_{k}\left(u\left(t_{k}\right)\right) .
$$

Applying the second boundary condition, one has

$$
\begin{aligned}
u(1)= & \frac{1}{\alpha-\int_{0}^{1} g(s) \Delta s} \int_{0}^{1} g(s)\left(\int_{s}^{1} \phi_{q}\left(\int_{0}^{\tau} f(r, u(r)) \nabla r\right) \Delta \tau\right) \Delta s \\
& +\frac{1}{\alpha-\int_{0}^{1} g(s) \Delta s}\left[\beta \phi_{q}\left(\int_{0}^{1} f(s, u(s)) \nabla s\right)+\int_{0}^{1} g(s) \sum_{t \leq t_{k}} I_{k}\left(u\left(t_{k}\right)\right) \Delta s\right] .
\end{aligned}
$$

Therefore, by (3.2) and (3.3), we have

$$
\begin{aligned}
u(t)= & \frac{1}{\alpha-\int_{0}^{1} g(s) \Delta s} \int_{0}^{1} g(s)\left(\int_{s}^{1} \phi_{q}\left(\int_{0}^{\tau} f(r, u(r)) \nabla r\right) \Delta \tau\right) \Delta s \\
& +\frac{1}{\alpha-\int_{0}^{1} g(s) \Delta s}\left[\beta \phi_{q}\left(\int_{0}^{1} f(s, u(s)) \nabla s\right)\right. \\
& \left.+\int_{0}^{1} g(s)\left(\sum_{s \leq t_{k}} I_{k}\left(u\left(t_{k}\right)\right)\right) \Delta s\right] \\
& +\int_{t}^{1} \phi_{q}\left(\int_{0}^{s} f(r, u(r)) \nabla r\right) \Delta s+\sum_{t \leq t_{k}} I_{k}\left(u\left(t_{k}\right)\right) .
\end{aligned}
$$

Then the proof of the sufficient part is complete.

Conversely, let $u$ be as in (3.1). Taking the delta derivative of $u(t)$ gives

$$
u^{\Delta}(t)=-\phi_{q}\left(\int_{0}^{t} f(s, u(s)) \nabla s\right), \quad \text { i.e., } \phi_{p}\left(u^{\Delta}(t)\right)=-\int_{0}^{t} f(s, u(s)) \nabla s .
$$

So $u^{\Delta}(0)=0$. Also, it is easy to see that $u(t)$ satisfies (1.2) and (1.3). Furthermore, from (H1)-(H3), and (3.1), it is clear that $u(t) \geq 0$. So, the proof of lemma is completed.

Lemma 3.2 If $u \in K$, then $\min _{t \in[0,1]_{\mathbb{T}}} u(t) \geq \gamma\|u\|$, where $\gamma=\frac{\int_{0}^{1} g(s)(1-s) \Delta s}{\alpha-\int_{0}^{1} g(s) s \Delta s}$.

Proof For $u \in K$, nonnegative and nonincreasing, we have

$$
\|u\|=u(0), \quad \min _{t \in[0,1]_{\mathbb{T}}} u(t)=u(1) .
$$

On the other hand, $u^{\Delta}(t)$ is nonincreasing on $J^{\prime}$. So, for every $t \in[0,1]_{\mathbb{T}}$, we have

$$
\frac{u(t)-u(1)}{1-t} \geq \frac{u(0)-u(1)}{1}
$$

i.e., $u(t) \geq(1-t) u(0)+t u(1)$.

Therefore,

$$
\int_{0}^{1} g(s) u(s) \Delta s \geq \int_{0}^{1} g(s)(1-s) u(0) \Delta s+\int_{0}^{1} g(s) s u(1) \Delta s .
$$


This, together with $\alpha u(1)+\beta u^{\Delta}(1)=\int_{0}^{1} g(s) u(s) \Delta s$, implies that

$$
u(1) \geq \frac{\int_{0}^{1} g(s)(1-s) \Delta s}{\alpha-\int_{0}^{1} g(s) s \Delta s} u(0) .
$$

So, the proof of the lemma is completed.

Define $T: K \rightarrow \mathbb{B}$ by

$$
\begin{aligned}
(T u)(t)= & \frac{1}{\alpha-\int_{0}^{1} g(s) \Delta s} \int_{0}^{1} g(s)\left(\int_{s}^{1} \phi_{q}\left(\int_{0}^{\tau} f(r, u(r)) \nabla r\right) \Delta \tau\right) \Delta s \\
& +\frac{1}{\alpha-\int_{0}^{1} g(s) \Delta s}\left[\beta \phi_{q}\left(\int_{0}^{1} f(s, u(s)) \nabla s\right)\right. \\
& \left.+\int_{0}^{1} g(s)\left(\sum_{s \leq t_{k}} I_{k}\left(u\left(t_{k}\right)\right)\right) \Delta s\right] \\
& +\int_{t}^{1} \phi_{q}\left(\int_{0}^{s} f(r, u(r)) \nabla r\right) \Delta s+\sum_{t \leq t_{k}} I_{k}\left(u\left(t_{k}\right)\right) .
\end{aligned}
$$

From (3.5) and Lemma 3.1, it is easy to obtain the following result.

Lemma 3.3 Assume that (H1)-(H3) hold. Then $T: K \rightarrow K$ is completely continuous.

Now we consider the existence of at least two positive solutions for the impulsive boundary value problem (1.1)-(1.3) by the fixed point theorem in [22]. Let us define the increasing, nonnegative, continuous functionals $\gamma, \beta$, and $\alpha$ on $K$ by

$$
\begin{aligned}
& \gamma(u)=\min _{t \in[0, \xi]_{\mathbb{T}}} u(t)=u(\xi), \\
& \beta(u)=\max _{t \in[\xi, \eta]_{\mathbb{T}}} u(t)=u(\xi), \\
& \alpha(u)=\max _{t \in[0, \eta]_{\mathbb{T}}} u(t)=u(0) .
\end{aligned}
$$

It is obvious that for each $u \in K$,

$$
\gamma(u) \leq \beta(u) \leq \alpha(u)
$$

In addition, by Lemma 3.2, for each $u \in K$,

$$
\|u\| \leq \frac{1}{\gamma} \min _{t \in[0,1]_{\mathbb{T}}} u(t) \leq \frac{1}{\gamma} \min _{t \in[0, \xi]_{\mathbb{T}}} u(t)=\frac{1}{\gamma} \gamma(u) .
$$

Thus,

$$
\|u\| \leq \frac{1}{\gamma} \gamma(u), \quad \forall u \in K .
$$


For the convenience, we denote

$$
\begin{aligned}
& A=(1-\xi) \phi_{q}(\xi), \\
& B=\frac{(m+1) \alpha+\beta}{\alpha-\int_{0}^{1} g(s) \Delta s}-\xi .
\end{aligned}
$$

Theorem 3.1 Suppose the assumptions of $(\mathrm{H} 1)-(\mathrm{H} 3)$ are satisfied. Let there exist positive numbers $a<b<c$ such that

$$
0<a<\frac{A}{B} b<\frac{A \gamma}{B} c,
$$

and assume that $f$ satisfies the following conditions:

(H4) $f(t, u)>\phi_{p}\left(\frac{c}{A}\right)$, for all $(t, u) \in[0, \xi]_{\mathbb{T}} \times\left[c, \frac{1}{\gamma} c\right]$,

(H5) $f(t, u)<\phi_{p}\left(\frac{b}{B}\right), \sup \left\{I_{j}(u)\right\}<\frac{b}{B}, j=1,2, \ldots, m$, for all $(t, u) \in[0,1]_{\mathbb{T}} \times\left[0, \frac{1}{\gamma} b\right]$,

(H6) $f(t, u)>\phi_{p}\left(\frac{a}{A}\right)$, for all $(t, u) \in[0,1]_{\mathbb{T}} \times[0, a]$.

Then the boundary value problem (1.1)-(1.3) has at least two positive solutions $u_{1}$ and $u_{2}$ satisfying

$$
a<\alpha\left(u_{1}\right) \quad \text { with } \beta\left(u_{1}\right)<b,
$$

and

$$
b<\beta\left(u_{2}\right) \quad \text { with } \gamma\left(u_{2}\right)<c .
$$

Proof We define the completely continuous operator $T$ by (3.5). Thus, it is easy to check that $T: \overline{K(\gamma, c)} \rightarrow K$.

We now show that all the conditions of Theorem 2.1 are satisfied. In order to show that condition (i) of Theorem 2.1, we choose $u \in \partial K(\gamma, c)$. Then $\gamma(u)=\min _{t \in[0, \xi]_{\mathbb{T}}} u(t)=$ $u(\xi)=c$, this implies that $c \leq u(t)$ for $t \in[0, \xi]_{\mathbb{T}}$. We recall that $\|u\| \leq \frac{1}{\gamma} \gamma(u)=\frac{1}{\gamma} c$. So, we have

$$
c \leq u(t) \leq \frac{1}{\gamma} c, \quad t \in[0, \xi]_{\mathbb{T}} .
$$

Then assumption (H4) implies

$$
f(t, u)>\phi_{p}\left(\frac{c}{a}\right), \quad \text { for all }(t, u) \in[0, \xi]_{\mathbb{T}} \times\left[c, \frac{1}{\gamma} c\right] .
$$

Therefore,

$$
\begin{aligned}
\gamma(T u)= & \min _{t \in[0, \xi]_{\mathbb{T}}}(T u)(t)=(T u)(\xi) \\
= & \frac{1}{\alpha-\int_{0}^{1} g(s) \Delta s}\left[\int_{0}^{1} g(s)\left(\int_{s}^{1} \phi_{q}\left(\int_{0}^{\tau} f(r, u(r)) \nabla r\right) \Delta \tau\right) \Delta s\right. \\
& \left.+\beta \phi_{q}\left(\int_{0}^{1} f(s, u(s)) \nabla s\right)\right]+\int_{\xi}^{1} \phi_{q}\left(\int_{0}^{s} f(r, u(r)) \nabla r\right) \Delta s \\
& +\frac{1}{\alpha-\int_{0}^{1} g(s) \Delta s} \int_{0}^{1} g(s)\left(\sum_{s \leq t_{k}} I_{k}\left(u\left(t_{k}\right)\right)\right) \Delta s+\sum_{t \leq t_{k}} I_{k}\left(u\left(t_{k}\right)\right)
\end{aligned}
$$




$$
\begin{aligned}
& \geq \int_{\xi}^{1} \phi_{q}\left(\int_{0}^{s} f(r, u(r)) \nabla r\right) \Delta s \geq \int_{\xi}^{1} \phi_{q}\left(\int_{0}^{\xi} f(r, u(r)) \nabla r\right) \Delta s \\
& =(1-\xi) \phi_{q}\left(\int_{0}^{\xi} f(r, u(r)) \nabla r\right) \\
& >(1-\xi) \phi_{q}(\xi) \frac{c}{A}=c .
\end{aligned}
$$

Hence, condition (i) is satisfied.

Secondly, we show that (ii) of Theorem 2.1 is satisfied. For this, we take $u \in \partial K(\beta, b)$. Then $\beta(u)=\max _{t \in[\xi, \eta]_{\mathbb{T}}} u(t)=u(\xi)=b$, this means $0 \leq u(t) \leq b$, for all $t \in[\xi, 1]_{\mathbb{T}}$. Noticing that $\|u\| \leq \frac{1}{\gamma} \gamma(u)=\frac{1}{\gamma} \beta(u)=\frac{1}{\gamma} b$, we get

$$
0 \leq u(t) \leq \frac{1}{\gamma} b, \quad \text { for } t \in[0,1]_{\mathbb{T}} .
$$

Then assumption (H5) implies

$$
f(t, u)<\phi_{p}\left(\frac{b}{B}\right), \quad \sup \left\{I_{j}(u)\right\}<\frac{b}{B} .
$$

Therefore

$$
\begin{aligned}
\beta(T u)= & \max _{t \in[\xi, \eta]_{\mathbb{T}}}(T u)(t)=(T u)(\xi) \\
= & \frac{1}{\alpha-\int_{0}^{1} g(s) \Delta s}\left[\int_{0}^{1} g(s)\left(\int_{s}^{1} \phi_{q}\left(\int_{0}^{\tau} f(r, u(r)) \nabla r\right) \Delta \tau\right) \Delta s\right. \\
& \left.+\beta \phi_{q}\left(\int_{0}^{1} f(s, u(s)) \nabla s\right)\right]+\int_{\xi}^{1} \phi_{q}\left(\int_{0}^{s} f(r, u(r)) \nabla r\right) \Delta s \\
& +\frac{1}{\alpha-\int_{0}^{1} g(s) \Delta s} \int_{0}^{1} g(s)\left(\sum_{s \leq t_{k}} I_{k}\left(u\left(t_{k}\right)\right)\right) \Delta s+\sum_{t \leq t_{k}} I_{k}\left(u\left(t_{k}\right)\right) \\
\leq & \frac{1}{\alpha-\int_{0}^{1} g(s) \Delta s}\left[\int_{0}^{1} g(s)\left(\int_{0}^{1} \phi_{q}\left(\int_{0}^{1} f(r, u(r)) \nabla r\right) \Delta \tau\right) \Delta s\right. \\
& \left.+\beta \phi_{q}\left(\int_{0}^{1} f(s, u(s)) \nabla s\right)\right]+\int_{\xi}^{1} \phi_{q}\left(\int_{0}^{1} f(r, u(r)) \nabla r\right) \Delta s \\
& +\frac{1}{\alpha-\int_{0}^{1} g(s) \Delta s} \int_{0}^{1} g(s)\left(\sum_{k=1}^{m} I_{k}\left(u\left(t_{k}\right)\right)\right) \Delta s+\sum_{k=1}^{m} I_{k}\left(u\left(t_{k}\right)\right) \\
= & {\left[\frac{\alpha+\beta}{\alpha-\int_{0}^{1} g(s) \Delta s}-\xi\right] \phi_{q}\left(\int_{0}^{1} f(r, u(r)) \nabla r\right)+\frac{\alpha}{\alpha-\int_{0}^{1} g(s) \Delta s} \sum_{k=1}^{m} I_{k}\left(u\left(t_{k}\right)\right) } \\
< & {\left[\frac{\alpha+\beta}{\alpha-\int_{0}^{1} g(s) \Delta s}-\xi\right] \frac{b}{B}+\frac{\alpha}{\alpha-\int_{0}^{1} g(s) \Delta s} m \frac{b}{B} } \\
= & \frac{b}{B}\left[\frac{(m+1) \alpha+\beta}{\alpha-\int_{0}^{1} g(s) \Delta s}-\xi\right] \\
= & b .
\end{aligned}
$$

So, we get $\beta(T u)<b$. Hence, condition (ii) is satisfied. 
Finally, we show that condition (iii) of Theorem 2.1 is satisfied. We note that $u(t)=\frac{2}{3} a$, $t \in[0,1]_{\mathbb{T}}$ is a member of $K(\alpha, a)$, and so $K(\alpha, a) \neq \emptyset$.

Now, let $u \in \partial K(\alpha, a)$. Then $\alpha(u)=\max _{t \in[0, \eta]_{\mathbb{T}}} u(t)=u(0)=a$. This implies $0 \leq u(t) \leq a$, $t \in[0,1]_{\mathbb{T}}$. Then we get

$$
0 \leq u(t) \leq a, \quad t \in[0,1]_{\mathbb{T}} .
$$

By assumption (H7),

$$
f(t, u)>\phi_{p}\left(\frac{a}{A}\right)
$$

Then

$$
\begin{aligned}
\alpha(T u) & =\max _{t \in[0, \eta]_{\mathbb{T}}}(T u)(t)=(T u)(0) \\
& \geq \int_{0}^{1} \phi_{q}\left(\int_{0}^{s} f(r, u(r)) \nabla r\right) \Delta s \geq \int_{\xi}^{1} \phi_{q}\left(\int_{0}^{s} f(r, u(r)) \nabla r\right) \Delta s \\
& \geq(1-\xi) \phi_{q}\left(\int_{0}^{\xi} f(r, u(r)) \nabla r\right) \\
& >(1-\xi) \frac{a}{A} \phi_{q}\left(\int_{0}^{\xi} \nabla r\right) \\
& =(1-\xi) \frac{a}{A} \phi_{q}(\xi)=a .
\end{aligned}
$$

So, we get $\alpha(T u)>a$. Thus, (iii) of Theorem 2.1 is satisfied. Hence, the impulsive boundary value problem (1.1)-(1.3) has at least two positive solutions $u_{1}$ and $u_{2}$ satisfying

$a<\alpha\left(u_{1}\right) \quad$ with $\beta\left(u_{1}\right)<b$,

and

$$
b<\beta\left(u_{2}\right) \quad \text { with } \gamma\left(u_{2}\right)<c .
$$

The proof is complete.

Next we consider the existence of at least two positive solutions for the BVP (1.1)-(1.3) by the fixed point theorem in [21].

We define

$$
\begin{aligned}
& K_{\rho}=\{u \in K:\|u\|<\rho\}, \\
& \Omega_{\rho}=\left\{u \in K: \min _{t \in[0,1]_{\mathbb{T}}} u(t)<\gamma \rho\right\}=\left\{u \in K: \gamma\|u\| \leq \min _{t \in[0,1]_{\mathbb{T}}} u(t)<\gamma \rho\right\} .
\end{aligned}
$$

The following results are proved in [21] (Lemma 2.5, p.693). 
Lemma 3.4 $\Omega_{\rho}$ has the following properties:

(a) $\Omega_{\rho}$ is open relative to $K$.

(b) $K_{\gamma \rho} \subset \Omega_{\rho} \subset K_{\rho}$.

(c) $u \in \Omega_{\rho}$ if and only if $\min _{t \in[0,1]_{\mathbb{T}}} u(t)=\gamma \rho$.

(d) If $u \in \Omega_{\rho}$, then $\gamma \rho \leq u(t) \leq \rho$ for $t \in[0,1]_{\mathbb{T}}$.

Now for convenience we introduce the following notations. Let

$$
\begin{aligned}
& f_{\gamma \rho}^{\rho}=\min \left\{\min _{t \in[0,1]_{\mathbb{T}}} \frac{f(t, u)}{\phi_{p}(\rho)}: u \in[\gamma \rho, \rho]\right\}, \\
& f_{0}^{\rho}=\max \left\{\max _{t \in[0,1]_{\mathbb{T}}} \frac{f(t, u)}{\phi_{p}(\rho)}: u \in[0, \rho]\right\}, \\
& I_{0}^{\rho}(k)=\max \left\{I_{k}(u): u \in[0, \rho]\right\}, \\
& \frac{1}{l}=\frac{(m+1) \alpha+\beta}{\alpha-\int_{0}^{1} g(s) \Delta s}, \quad \frac{1}{L}=\frac{\beta}{\alpha-\int_{0}^{1} g(s) \Delta s} .
\end{aligned}
$$

Theorem 3.2 Suppose (H1) and (H3) hold.

(H7) There exist $\rho_{1}, \rho_{2}, \rho_{3} \in(0, \infty)$ with $\rho_{1}<\gamma \rho_{2}$ and $\rho_{2}<\rho_{3}$ such that

$$
f_{0}^{\rho_{1}}<\phi_{p}(l), \quad I_{0}^{\rho_{1}}(k)<l \rho_{1}, \quad f_{\gamma \rho_{2}}^{\rho_{2}}>\phi_{p}(L), \quad f_{0}^{\rho_{3}}<\phi_{p}(l), \quad I_{0}^{\rho_{3}}(k)<l \rho_{3} .
$$

Then problem (1.1)-(1.3) has at least two positive solutions $u_{1}, u_{2}$ with $u_{1} \in \Omega_{\rho_{2}} \backslash \bar{K}_{\rho_{1}}, u_{2} \in K_{\rho_{3}} \backslash \bar{\Omega}_{\rho_{2}}$.

(H8) There exist $\rho_{1}, \rho_{2}, \rho_{3} \in(0, \infty)$ with $\rho_{1}<\rho_{2}<\gamma \rho_{3}<\rho_{3}$ such that

$$
f_{\gamma \rho_{1}}^{\rho_{1}}>\phi_{p}(L), \quad f_{0}^{\rho_{2}}<\phi_{p}(l), \quad I_{0}^{\rho_{2}}(k)<l \rho_{2}, \quad f_{\gamma \rho_{3}}^{\rho_{3}}>\phi_{p}(L) .
$$

Then problem (1.1)-(1.3) has at least two positive solutions $u_{1}, u_{2}$ with $u_{1} \in K_{\rho_{2}} \backslash \bar{\Omega}_{\rho_{1}}, u_{2} \in \Omega_{\rho_{3}} \backslash \bar{K}_{\rho_{2}}$.

Proof We only consider condition (H7). If (H8) holds, then the proof is similar to that of the case when (H7) holds. By Lemma 3.3, we know that the operator $T: K \rightarrow K$ is completely continuous.

First, we show that $i_{K}\left(T, K_{\rho_{1}}\right)=1$. In fact, by $(3.5), f_{0}^{\rho_{1}}<\phi_{p}(l)$, and $I_{0}^{\rho_{1}}(k)<l \rho_{1}$, we have, for $u \in \partial K_{\rho_{1}}$,

$$
\begin{aligned}
(T u)(t)= & \frac{1}{\alpha-\int_{0}^{1} g(s) \Delta s} \int_{0}^{1} g(s)\left(\int_{s}^{1} \phi_{q}\left(\int_{0}^{\tau} f(r, u(r)) \nabla r\right) \Delta \tau\right) \Delta s \\
& +\frac{1}{\alpha-\int_{0}^{1} g(s) \Delta s}\left[\beta \phi_{q}\left(\int_{0}^{1} f(s, u(s)) \nabla s\right)\right. \\
& \left.+\int_{0}^{1} g(s)\left(\sum_{s \leq t_{k}} I_{k}\left(u\left(t_{k}\right)\right)\right) \Delta s\right]+\int_{t}^{1} \phi_{q}\left(\int_{0}^{s} f(r, u(r)) \nabla r\right) \Delta s \\
& +\sum_{t \leq t_{k}} I_{k}\left(u\left(t_{k}\right)\right)
\end{aligned}
$$




$$
\begin{aligned}
& \leq \frac{1}{\alpha-\int_{0}^{1} g(s) \Delta s} \int_{0}^{1} g(s)\left(\int_{0}^{1} \phi_{q}\left(\int_{0}^{\tau} f(r, u(r)) \nabla r\right) \Delta \tau\right) \Delta s \\
& +\frac{1}{\alpha-\int_{0}^{1} g(s) \Delta s}\left[\beta \phi_{q}\left(\int_{0}^{1} f(s, u(s)) \nabla s\right)\right. \\
& \left.+\int_{0}^{1} g(s)\left(\sum_{k=1}^{m} I_{k}\left(u\left(t_{k}\right)\right)\right) \Delta s\right]+\int_{0}^{1} \phi_{q}\left(\int_{0}^{s} f(r, u(r)) \nabla r\right) \Delta s \\
& +\sum_{k=1}^{m} I_{k}\left(u\left(t_{k}\right)\right) \\
& =\frac{\alpha}{\alpha-\int_{0}^{1} g(s) \Delta s}\left[\int_{0}^{1} \phi_{q}\left(\int_{0}^{s} f(r, u(r)) \nabla r\right) \Delta s+\sum_{k=1}^{m} I_{k}\left(u\left(t_{k}\right)\right)\right] \\
& +\frac{\beta}{\alpha-\int_{0}^{1} g(s) \Delta s} \phi_{q}\left(\int_{0}^{1} f(s, u(s)) \nabla s\right) \\
& <\frac{\beta+\alpha}{\alpha-\int_{0}^{1} g(s) \Delta s} \phi_{q}\left(\int_{0}^{1} f(s, u(s)) \nabla s\right)+\frac{\alpha \sum_{k=1}^{m} I_{k}\left(u\left(t_{k}\right)\right)}{\alpha-\int_{0}^{1} g(s) \Delta s} \\
& <\frac{\beta+\alpha}{\alpha-\int_{0}^{1} g(s) \Delta s} l \rho_{1}+\frac{\alpha}{\alpha-\int_{0}^{1} g(s) \Delta s} l \rho_{1} m \\
& =l \rho_{1}\left[\frac{\beta+(m+1) \alpha}{\alpha-\int_{0}^{1} g(s) \Delta s}\right]=\rho_{1},
\end{aligned}
$$

i.e., $\|T u\|<\|u\|$ for $u \in \partial K_{\rho_{1}}$. By (i) of Theorem 2.2, we obtain $i_{K}\left(T, K_{\rho_{1}}\right)=1$.

Secondly, we show that $i_{K}\left(T, \Omega_{\rho_{2}}\right)=0$. Let $e(t) \equiv 1$. Then $e \in \partial K_{1}$. We claim that

$$
u \neq T u+B e, \quad u \in \partial \Omega_{\rho_{2}}, B>0 .
$$

Suppose that there exist $u_{0} \in \partial \Omega_{\rho_{2}}$ and $B_{0}>0$ such that

$$
u_{0}=T u_{0}+B_{0} e
$$

Then Lemma 3.1, Lemma 3.2, and (3.6) imply that for $t \in[0,1]$

$$
\begin{aligned}
u_{0} & =T u_{0}+B_{0} e \geq \gamma\left\|T u_{0}\right\|+B_{0} \\
& \geq \gamma \frac{\beta}{\alpha-\int_{0}^{1} g(s) \Delta s} \phi_{q}\left(\int_{0}^{1} f(s, u(s)) \nabla s\right)+B_{0} \\
& >\gamma \frac{\beta}{\alpha-\int_{0}^{1} g(s) \Delta s} \phi_{q}\left(\int_{0}^{1} \phi_{p}\left(L \rho_{2}\right) \nabla s\right)+B_{0} \\
& =\gamma \frac{\beta}{\alpha-\int_{0}^{1} g(s) \Delta s} L \rho_{2}+B_{0}=\gamma \rho_{2}+B_{0},
\end{aligned}
$$

i.e. $\gamma \rho_{2}>\gamma \rho_{2}+B_{0}$, which is a contradiction. Hence by (ii) of Theorem 2.2, it follows that $i_{K}\left(T, \Omega_{\rho_{2}}\right)=0$.

Finally, similar to the proof of $i_{K}\left(T, K_{\rho_{1}}\right)=1$, we can prove that $i_{K}\left(T, K_{\rho_{3}}\right)=1$. Since $\rho_{1}<$ $\gamma \rho_{2}$ and Lemma 3.4(b), we have $\bar{K}_{\rho_{1}} \subset K_{\gamma \rho_{2}} \subset \Omega_{\rho_{2}}$. Similarly to $\rho_{2}<\rho_{3}$ and Lemma 3.4(b), 
we have $\bar{\Omega}_{\rho_{2}} \subset K_{\rho_{2}} \subset K_{\rho_{3}}$. Therefore (iii) of Theorem 2.2 implies that BVP (1.1)-(1.3) has at least two positive solutions $u_{1}, u_{2}$ with $u_{1} \in \Omega_{\rho_{2}} \backslash \bar{K}_{\rho_{1}}, u_{2} \in K_{\rho_{3}} \backslash \bar{\Omega}_{\rho_{2}}$.

Theorem 3.2 can be generalized to obtain many solutions.

Theorem 3.3 Suppose $(\mathrm{H} 1)-(\mathrm{H} 3)$ hold. Then we have the following assertions.

(H9) There exists $\left\{\rho_{i}\right\}_{i=1}^{2 m_{0}+1} \subset(0, \infty)$ with $\rho_{1}<\gamma \rho_{2}<\rho_{2}<\rho_{3}<\gamma \rho_{4}<\cdots<\gamma \rho_{2 m_{0}}<$ $\rho_{2 m_{0}}<\rho_{2 m_{0}+1}$ such that

$$
\begin{aligned}
& f_{0}^{\rho_{2 m-1}}<\phi_{p}(l), \quad I_{0}^{\rho_{2 m-1}}<l \rho_{2 m-1} \quad\left(m=1,2, \ldots, m_{0}, m_{0}+1\right), \\
& f_{\gamma \rho_{2} m}^{\rho_{2} m}>\phi_{p}(L) \quad\left(m=1,2, \ldots, m_{0}\right) .
\end{aligned}
$$

Then problem (1.1)-(1.3) has at least $2 m_{0}$ solutions in $K$.

(H10) There exists $\left\{\rho_{i}\right\}_{i=1}^{2 m_{0}} \subset(0, \infty)$ with $\rho_{1}<\gamma \rho_{2}<\rho_{2}<\rho_{3}<\gamma \rho_{4}<\cdots<\gamma \rho_{2 m_{0}}<\rho_{2 m_{0}}$ such that

$$
f_{0}^{\rho_{2 m-1}}<\phi_{p}(l), \quad I_{0}^{\rho_{2 m-1}}<l \rho_{2 m-1}, \quad f_{\gamma \rho_{2} m}^{\rho_{2} m}>\phi_{p}(L) \quad\left(m=1,2, \ldots, m_{0}\right) .
$$

Then problem (1.1)-(1.3) has at least $2 m_{0}-1$ solutions in $K$.

Theorem 3.4 Suppose (H1)-(H3) hold. Then we have the following assertions.

(H11) There exists $\left\{\rho_{i}\right\}_{i=1}^{2 m_{0}+1} \subset(0, \infty)$ with $\rho_{1}<\rho_{2}<\gamma \rho_{3}<\rho_{3}<\cdots<\rho_{2 m_{0}}<\gamma \rho_{2 m_{0}+1}<$ $\rho_{2 m_{0}+1}$ such that

$$
\begin{aligned}
& f_{\gamma \rho_{2 m-1}}^{\rho_{2 m-1}}>\phi_{p}(L) \quad\left(m=1,2, \ldots, m_{0}, m_{0}+1\right), \quad f_{0}^{\rho_{2} m}<\phi_{p}(l), \\
& I_{0}^{\rho_{2 m}}<l \rho_{2 m} \quad\left(m=1,2, \ldots, m_{0}\right) .
\end{aligned}
$$

Then problem (1.1)-(1.3) has at least $2 m_{0}$ solutions in $K$.

(H12) There exists $\left\{\rho_{i}\right\}_{i=1}^{2 m_{0}} \subset(0, \infty)$ with $\rho_{1}<\rho_{2}<\gamma \rho_{3}<\rho_{3}<\cdots<\gamma \rho_{2 m_{0}-1}<\rho_{2 m_{0}-1}<$ $\rho_{2 m_{0}}$ such that

$$
f_{\gamma \rho_{2 m-1}}^{\rho_{2 m-1}}>\phi_{p}(L), \quad f_{0}^{\rho_{2 m}}<\phi_{p}(l), \quad I_{0}^{\rho_{2 m}}<l \rho_{2 m} \quad\left(m=1,2, \ldots, m_{0}\right) .
$$

Then problem (1.1)-(1.3) has at least $2 m_{0}-1$ solutions in $K$.

\section{Example}

Example 4.1 In BVP (1.1)-(1.3), suppose that $m=1, p=3, t_{1}=\frac{1}{3}, g(s)=s, \alpha=\frac{3}{2}, \beta=\frac{1}{2}$, and $\mathbb{T}=\left\{\frac{n+1}{4}: n \in \mathbb{N}\right\} \cup[2,5]$, i.e.

$$
\begin{aligned}
& -\left[\phi_{3}\left(u^{\Delta}(t)\right)\right]^{\nabla}=f(t, u(t)), \quad t \in[0,1]_{\mathbb{T}} \backslash\left\{\frac{1}{3}\right\}, \\
& -\left(u\left(\frac{1}{3}^{+}\right)-u\left(\frac{1^{-}}{3}\right)\right)=I_{1}\left(u\left(\frac{1}{3}\right)\right), \\
& u^{\Delta}(0)=0, \quad \frac{3}{2} u(1)+\frac{1}{2} u^{\Delta}(1)=\int_{0}^{1} s u(s) \Delta s,
\end{aligned}
$$


where

$$
\begin{aligned}
& f(t, u)= \begin{cases}\frac{1}{25}(-u+2), & u \in[0,2] ; \\
2,838(u-2), & u \in(2,5] ; \\
8,514, & u \in(5, \infty) ;\end{cases} \\
& I_{1}(u)=\frac{u}{8} .
\end{aligned}
$$

Taking $\rho_{1}=2, \rho_{2}=41, \rho_{3}=200$, we have $l=\frac{15}{28}, L=\frac{9}{4}, \gamma=\frac{5}{41}$. We obtain

$$
2=\rho_{1}<\gamma \rho_{2}=5 \text { and } 41=\rho_{2}<\rho_{3}=200 .
$$

Now, we show that $(\mathrm{H} 7)$ is satisfied:

$$
\begin{aligned}
& f_{0}^{2}=\frac{1}{50}<\phi_{3}\left(\frac{15}{28}\right)=\frac{225}{784}, \quad I_{0}^{2}=\frac{1}{4}<l \rho_{1}=\frac{15}{14}, \\
& f_{5}^{41}=\frac{8,514}{1,681}>\phi_{3}\left(\frac{9}{4}\right)=\frac{81}{16}, \\
& f_{0}^{200}=\frac{4,257}{20,000}<\phi_{3}\left(\frac{15}{28}\right)=\frac{225}{784} \text { and } I_{0}^{200}=25<l \rho_{3}=\frac{750}{7} .
\end{aligned}
$$

Then all conditions of Theorem 3.2 hold. Hence, we find that the BVP (4.1)-(4.3) has at least two positive solutions.

\section{Competing interests}

The authors declare that they have no competing interests.

\section{Authors' contributions}

All authors contributed equally to the manuscript and typed, read, and approved the final manuscript.

\section{Author details}

'Department of Mathematics, Ege University, Bornova, Izmir, 35100, Turkey. ㄹepartment of Mathematics, Gazi University, Teknikokullar, Ankara, 06500, Turkey.

\section{Acknowledgements}

The authors would like to thank the referees for their valuable suggestions and comments.

\section{Received: 5 October 2013 Accepted: 2 May 2014 Published: 15 May 2014}

\section{References}

1. Akhmet, M: Principles of Discontinuous Dynamical Systems. Springer, New York (2010)

2. Benchohra, M, Henderson, J, Ntouyas, S: Impulsive Differential Equations and Inclusions. Hindawi, New York (2006)

3. Lakshmikantham, V, Bainov, DD, Simeonov, PS: Theory of Impulsive Differential Equations. World Scientific, Singapore (1989)

4. Samoilenko, AM, Perestyuk, NA: Impulsive Differential Equations. World Scientific, Singapore (1995)

5. Bohner, M, Peterson, A: Dynamic Equations on Time Scales: An Introduction with Applications. Birkhäuser, Boston (2001)

6. Bohner, M, Peterson, A: Advances in Dynamic Equations on Time Scales. Birkhäuser, Boston (2003)

7. Chen, H, Wang, H, Zhang, Q, Zhou, T: Double positive solutions of boundary value problems for $p$-Laplacian impulsive functional dynamic equations on time scales. Comput. Math. Appl. 53, 1473-1480 (2007)

8. Chen, $\mathrm{H}$, Wang, $\mathrm{H}$ : Triple positive solutions of boundary value problems for $p$-Laplacian impulsive dynamic equations on time scales. Math. Comput. Model. 47, 917-924 (2008)

9. Jin, Y, Zhang, Z, Yang, J, Song, N: Positive solutions to boundary value problems for $m$ impulsive points $p$-Laplacian dynamic equations on time scales. J. Univ. Sci. Technol. China 41, 497-503 (2011)

10. Li, $\mathrm{P}, \mathrm{Chen}, \mathrm{H}, \mathrm{Wu}, \mathrm{Y}$ : Multiple positive solutions of $n$-point boundary value problems for $p$-Laplacian impulsive dynamic equations on time scales. Comput. Math. Appl. 60, 2572-2582 (2010) 
11. Liang, R, Shen, J: Triple positive solutions to bvp for $p$-Laplacian impulsive dynamic equations on time scales. Dyn. Contin. Discrete Impuls. Syst., Ser. A Math. Anal. 18, 719-730 (2011)

12. Benchohra, M, Ntouyas, SK, Ouahab, A: Extremal solutions of second order impulsive dynamic equations on time scales. J. Math. Anal. Appl. 32, 425-434 (2006)

13. Li, J, Shen, J: Existence results for second-order impulsive boundary value problems on time scales. Nonlinear Anal. 70, 1648-1655 (2009)

14. Xing, Y, Wang, Q, Chen, D: Antiperiodic boundary value problem for second-order impulsive differential equations on time scales. Adv. Differ. Equ. 2009, Article ID 567329 (2009)

15. Henderson, J: Double solutions of impulsive dynamic boundary value problems on a time scale. J. Differ. Equ. Appl. 8 , 345-356 (2002)

16. Feng, M, Du, B, Ge, W: Impulsive boundary value problems with integral boundary conditions and one-dimensional p-Laplacian. Nonlinear Anal. 70, 3119-3126 (2009)

17. Zhang, X, Ge, W: Impulsive boundary value problems involving the one-dimensional p-Laplacian. Nonlinear Anal. 70 1692-1701 (2009)

18. Liang, S, Zhang, J: The existence of countably many positive solutions for some nonlinear singular three-point impulsive boundary value problems. Nonlinear Anal. 71, 4588-4597 (2009)

19. LV, ZW, Liang, J, Xiao, TJ: Multiple positive solutions for second order impulsive boundary value problems in Banach spaces. Electron. J. Qual. Theory Differ. Equ. 38, 1-15 (2010)

20. Karaca, IY: On positive solutions for fourth-order boundary value problem with impulse. J. Comput. Appl. Math. 225 356-364 (2009)

21. Lan, KQ: Multiple positive solutions of semilinear differential equations with singularities. J. Lond. Math. Soc. 63 690-704 (2001)

22. Avery, Rl, Henderson, J: Two positive fixed points of nonlinear operators on ordered Banach spaces. Commun. Appl. Nonlinear Anal. 8, 27-36 (2001)

10.1186/1029-242X-2014-196

Cite this article as: Tokmak and Karaca: Existence of positive solutions for $p$-Laplacian impulsive boundary value problems on time scales. Journal of Inequalities and Applications 2014, 2014:196

\section{Submit your manuscript to a SpringerOpen ${ }^{\odot}$ journal and benefit from:}

- Convenient online submission

- Rigorous peer review

- Immediate publication on acceptance

Open access: articles freely available online

- High visibility within the field

- Retaining the copyright to your article 